EPJ Web of Conferences 110,01022 (2016)

DOI: $10.1051 /$ epjconf/201611001022

(C) Owned by the authors, published by EDP Sciences, 2016

\title{
SYNTHESIS OF ULTRADISPERSE CARBON DIOXIDE POWDER WITH PLASMA-DYNAMIC METHOD IN THE COAXIAL MAGNETO-PLASMA ACCELERATOR
}

\author{
Evgeniya. O. Golyanskaya ${ }^{1}$, Aleksandr A. Sivkov ${ }^{1}$, Zhanna S. Anikina ${ }^{1, a}$ \\ ${ }^{1}$ National Research Tomsk Polytechnic University, 634050 Tomsk, Russia
}

\begin{abstract}
One of the most promising trends in modern physics is the high-temperature superconductivity. Analysis of high-temperature superconductors revealed that almost all of them are complex copper-based oxides. Studies have shown the possibility of using them for the synthesis of coaxial magneto accelerator. Studies have identified the products synthesized soot: $\mathrm{Cu}, \mathrm{Cu} 2 \mathrm{O}, \mathrm{CuO}$, their shape and size. Also been deciphered and electron microscopy confirmed the composition of the nanopowder obtained in laboratory conditions.
\end{abstract}

\section{Introduction}

Today superconductivity is one of the most promising areas of physics, which generates interest of many scientists. However, practical application of high-temperature superconductors is largely limited by the technology.

Literature review shows that currently superconductivity in cuprates results from the copperoxygen layer where copper atoms form a square grid. Copper atoms are located at grid points, while oxygen atoms are on the lines connecting these points $[1,2]$. The history of superconductivity is a succeeding discovery of more and more complex structures. It all began with the synthesis of liquid helium, thereby opening the way to the systematic study of the material properties at temperatures close to absolute zero, when the material lose electrical resistance [3]. High-temperature superconductors were discovered more than 20 years ago, but still remain a mystery [4].

\section{Coaxial magneto-plasma accelerator}

To investigate the superconducting materials, ultrafine copper oxide powder was synthesized in the coaxial magneto-plasma accelerator designed by TPU scientists [5]. This accelerator technology can be used to accelerate plasma to hyperspeed.

${ }^{a}$ Corresponding author: janeanikina@yandex.ru

This is an Open Access article distributed under the terms of the Creative Commons Attribution License 4.0, which permits unrestricted use, distribution, and reproduction in any medium, provided the original work is properly cited. 


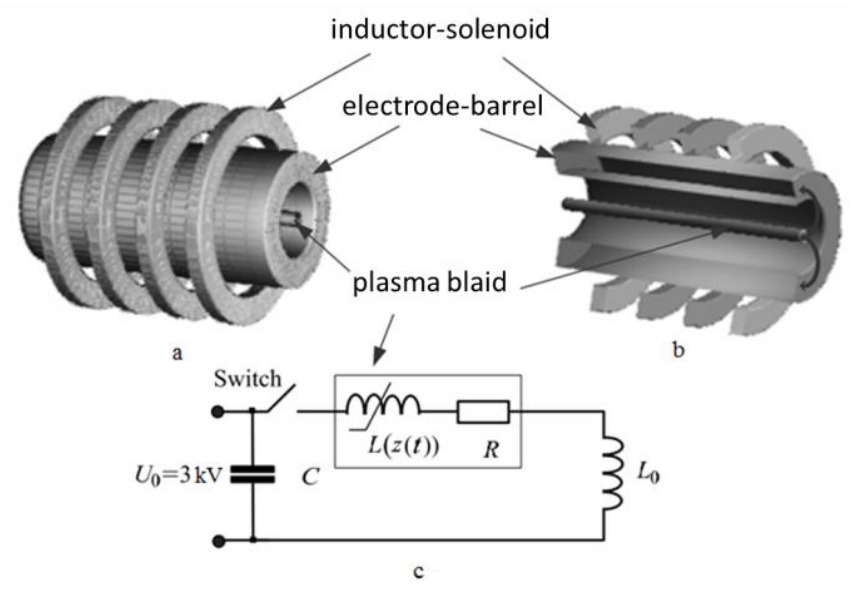

Figure 1. Simplified model of coaxial magneto-plasma accelerator: a) conductive part; b) cross-section; c) electrical circuit [6].

The accelerator is designed as a coaxial shaft-electrode system separated by an insulator and placed inside the solenoid. The shaft is cylinder-shaped. With the key closure, current flows from the capacitor bank via solenoid coils, passes through the shaft and central electrode to the capacitor through the switch. Arc discharge results from the insulator surface breakdown. Plasma is compressed by the intrinsic current magnetic field and by the solenoid magnetic field and is shaped as piston. The generated jet impinges into the reactor chamber, where the material is sputtered off the electrode surface, and nanosized particles are formed.

\section{Experiment}

\subsection{Experimental conditions}

During the experimental work, copper oxide powder was synthesized by means of coaxial magnetoplasma accelerator. The research has shown that the experimental conditions affect the resulting powder, the dimensions and properties of the nanoparticles. Conditions of the experiment are presented in Table.

Table. Experimental conditions for the synthesis of ultrafine copper oxide powder with coaxial magneto-plazma accelerator.

\begin{tabular}{|c|c|}
\hline Plasma & $\mathrm{Cu}$ \\
\hline Medium & Air \\
\hline Charging voltage & $3 \mathrm{kV}$ \\
\hline Capacity & $12 \mathrm{mF}$ \\
\hline Electrode & Steel + copper tip \\
\hline
\end{tabular}

\subsection{Output evaluation}

After the experiment, X-ray microscopy of the obtained ultrafine powders was made. PowderCell package and structural database PDF $4+$ were used for full-profile X-ray analysis. 


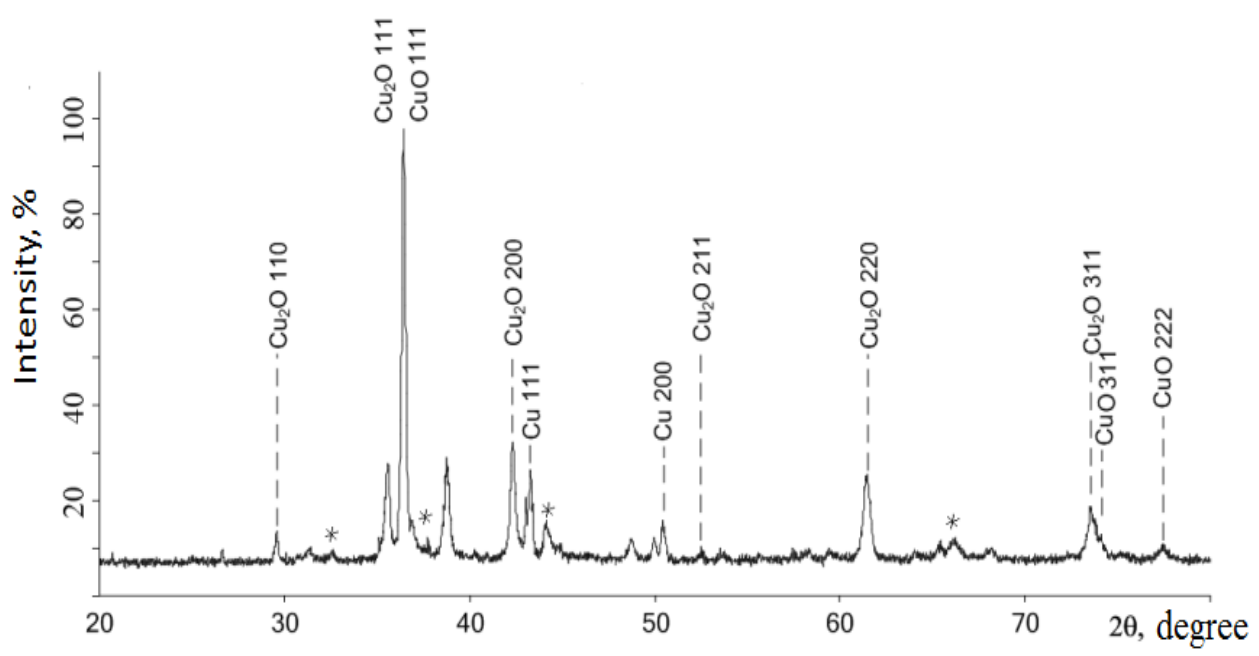

Figure 2. X-ray diffraction analysis.

The next synthesized powder phases were registered:

- copper oxide (I) (Cu2O) - 3,5\%

- copper oxide (II) $(\mathrm{CuO})$, exhibiting the highest rate (nearly $85 \%$ )

- $\quad$ pure copper $(\mathrm{Cu})$ (nearly $8 \%)$

- impurity phases, presented on radiographs as implicit peaks marked with an asterisk $\left(^{*}\right)$ - about $4 \%$. Their presence in the synthesized powder could be explained by the fact that the target material used in the experiment is aluminum. During the experiment, erosion occurred when melting in the plasma jet. Therefore, aluminum oxide $\left(\mathrm{Al}_{2} \mathrm{O}_{3}\right)$ is one of the fusion products.

Along with that, transmission electron microscopy data were also obtained and interpreted [7]. The results are shown in Figure 3.

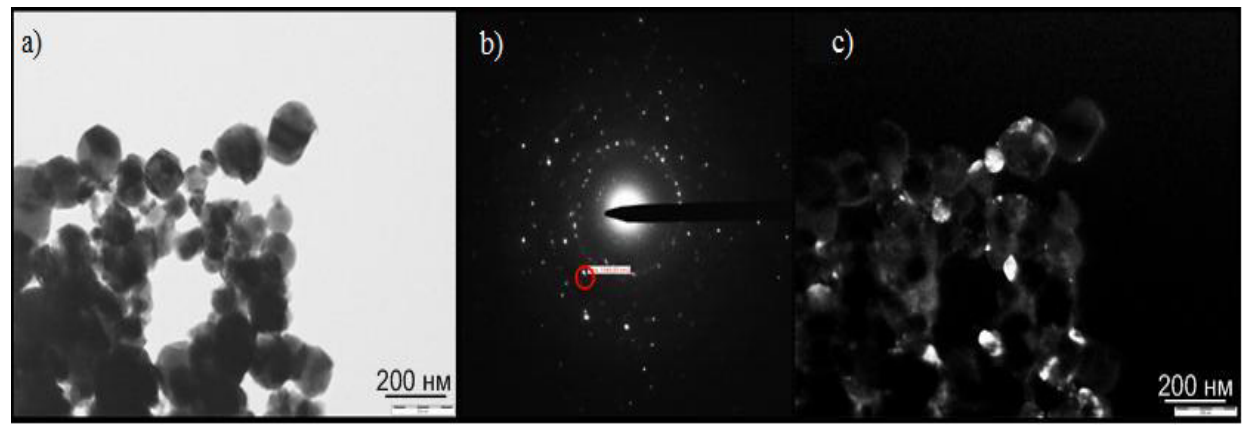

Figure 3. Transmission electron microscopy results: a) bright field image; b) electron diffraction pattern on the selected area; c) dark-field image.

Bright field image allowed to identify particle morphology. The particles form a convex polygon with rounded corners. Their size varies from 80 to $150 \mathrm{~nm}$. Lighter, circular shaped particles are copper oxides. Darker, angular shaped ones are copper.

According to the electron diffraction pattern for the selected area, it has been determined that the rubricated area is the crystallographic copper phase. Dark-field image was obtained when shifting the aperture diaphragm to the selected reflex point area representing crystallographic copper phase. As a result of the study, electron microscopy was interpreted. The composition of the nanopowder, obtained in laboratory conditions, was confirmed and its phases were defined. 


\section{Conclusion}

The practical task of our research was to obtain nanopowders with a small percentage of impurities and to increase the synthesis reproducibility. The method described meets these requirements. In future, we plan to obtain copper oxide using a coaxial magnetoplazma accelerator to synthesize complex high-temperature superconducting materials.

\section{References}

1. E. Demler, Sh. Zhang, Nature 396, 733 (1998)

2. S.G. Titova, D.O. Shorikov, V.F. Balakirev, T.S. Irvine John, I. Bryntse, Physica B, 284, (2000)

3. N.N. Bogolyubov, et al, New method in the theory of superconductivity (AN SSSR, Moscow, 1958)

4. Superconductors site - URL: http://www.superconductors.org

5. A.A. Sivkov, Coaxial accelerator. Patent RF 2150652 (2000)

6. A.A. Sivkov, Yu.N. Isaev, O.V. Vasil'eva, A.M. Kuptsov, Herald of TPU 4 (317), 33 (2010)

7. I.B. Borovskiy, Physical basics of X-ray spectral research (MGU, Moscow, 1956) 